\title{
High intraoperative endotracheal tube cuff pressure incidence and clinical impact
}

\author{
Abie H. Mendelsohn, MD $\odot \cdot$ Laith Mukdad, BA $\cdot$ Anahat Dhillon, MD
}

Received: 26 September 2017/Revised: 6 November 2017/ Accepted: 13 November 2017 / Published online: 4 December 2017

(C) Canadian Anesthesiologists' Society 2017

\section{To the Editor,}

Endotracheal intubation constitutes a routine function of anesthetic practice in the operating room, sealing the airway to prevent leaks and aspiration of pharyngeal contents into the trachea. However, the rate of laryngotracheal injury following intubation has been suggested to be $11 \%{ }^{1}$ It is theorized that the vast majority of post-intubation sequelae are related to persistently elevated cuff pressures. ${ }^{2}$

Although considerable attention is paid to the state of cuff pressure management for intubated patients admitted to intensive care units, ${ }^{3}$ almost no attention is given to the status of intraoperative cuff pressure management. To evaluate the potential for quality improvement, we aimed to identify the overall incidence of high intraoperative cuff pressure within a high-volume surgical academic center. Our University Office of the Human Protection Program policy regarding quality assurance indicated that Institutional Review Board approval was not required.

To measure the cuff pressure, we utilized an NS 60-PBS $60 \mathrm{cmH}_{2} \mathrm{O}$ Vacuum/Pressure Gauge (Instrumentation Industries, Inc., Bethel Park, PA, USA) connected via a three-way stopcock to the endotracheal tube pilot balloon. Specifically, the steps followed for cuff pressure measurements were:

A. H. Mendelsohn, MD ( $₫) \cdot$ L. Mukdad, BA

Department of Head and Neck Surgery, UCLA Voice Center for

Medicine and the Arts, David Geffen School of Medicine at

UCLA, Los Angeles, CA, USA

e-mail: AMendelsohn@mednet.ucla.edu

A. Dhillon, MD

Department of Anesthesiology, David Geffen School of

Medicine at UCLA, Los Angeles, CA, USA
1. Wash hands and put on gloves.

2. Ensure the stopcock "off" sign is in the direction of the valve that will attach to the pilot balloon circuit.

3. With a gloved finger, cover the remaining open valve.

4. Push the syringe inward until the pressure on the manometer reads approximately $25 \mathrm{cmH}_{2} \mathrm{O}$.

5. Attach the stopcock valve to the pilot balloon.

6. Turn the "off" sign $90^{\circ}$ to face the valve occluded by the gloved finger.

7. After momentary pressure adjustment, read and record the pressure level from the gauge face.

8. Immediately turn the "off" sign $90^{\circ}$ back in the direction of the pilot balloon.

9. Detach the stopcock.

Random sampling of active surgical cases defined the study group. Exclusion criteria included age less than $16 \mathrm{yr}$ and intraoperative use of nitrous oxide. Between 1 August and 15 October 2016, intraoperative cuff pressures of 156 patients were measured.

Median [interquartile range] cuff pressures of 35 [24-46] $\mathrm{cmH}_{2} \mathrm{O}$ were noted, a result above the acceptable range of 20-30 $\mathrm{cmH}_{2} \mathrm{O}$ reported in guidelines. ${ }^{4}$ Only 52 of the 156 (33\%) patients had cuff pressures between $20-30 \mathrm{cmH}_{2} \mathrm{O}$ (Figure). Cuff pressures greater than $30 \mathrm{cmH}_{2} \mathrm{O}$ were noted in 86 of $156(55 \%)$ patients from every surgical service, while only $18(12 \%)$ patients had a cuff pressure less than $20 \mathrm{cmH}_{2} \mathrm{O}$. Postoperative dysphagia was noted in $15(10 \%)$ patients, but our study was inadequately powered to identify associations with this outcome.

A major cause of intubation-related injury is overinflation and increased cuff pressure. ${ }^{1,2}$ Our quality improvement study clearly demonstrated a considerable incidence of high intraoperative cuff pressures throughout all surgical specialties. The fairly marked non-compliance 


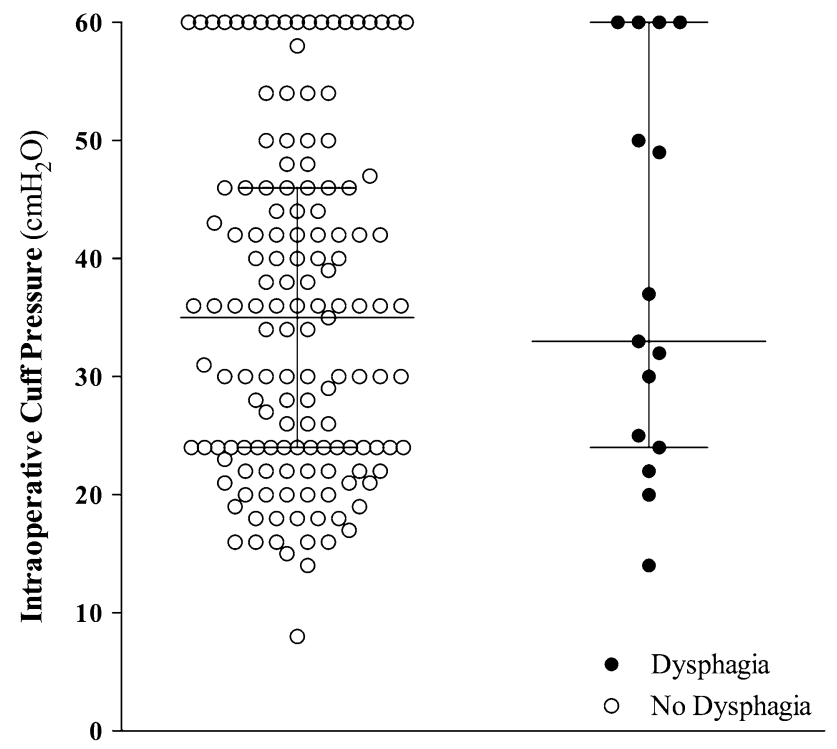

Figure Dot plot of all patients' endotracheal cuff pressures stratified by dysphagia diagnosis. The median [interquartile range (IQR)] cuff pressure of all patients was 34 [24-46] $\mathrm{cmH}_{2} \mathrm{O}$, with a median [IQR] in the 141 patients with no postoperative dysphagia of 35 [24-46] $\mathrm{cmH}_{2} \mathrm{O}$ and a median [IQR] in the 15 patients with postoperative dysphagia of 33 [24-60] $\mathrm{cmH}_{2} \mathrm{O}$

with standards common in critical care is an issue amenable to quality improvement.

Assessing cuff pressures is a low-cost and low riskeffort that requires approximately $30 \mathrm{sec}$ to perform. Training and practice of the measurement technique require approximately $15-20 \mathrm{~min}$. A theoretical risk of cuff deflation during measurement was quickly seen as a non-issue in our study with repeat measurements demonstrating no change in cuff pressure. Our data have influenced members of our institution's cardiothoracic, liver, and neurosurgical services who have begun regularly monitoring intraoperative cuff pressures. A large trial with long-term follow-up is required to define the potential benefits of cuff pressure management but is not required for anesthesiologists to adopt practices that are standard of care for our colleagues in critical care.

Conflicts of interest None declared.

Editorial responsibility This submission was handled by Dr. Gregory L. Bryson, Deputy Editor-in-Chief, Canadian Journal of Anesthesia.

Funding None.

\section{References}

1. Esteller-Moré E, Ibañez J, Matiñó E, Ademà JM, Nolla M, Quer $I M$. Prognostic factors in laryngotracheal injury following intubation and/or tracheotomy in ICU patients. Eur Arch Otorhinolaryngol 2005; 262: 880-3.

2. Bishop MJ. Mechanisms of laryngotracheal injury following prolonged tracheal intubation. Chest 1989; 96: 185-6.

3. Mahler F, Muheim MH, Intaglietta M, Bollinger A, Anliker M. Blood pressure fluctuations in human nailfold capillaries. Am J Physiol 1979; 236: H888-93.

4. Talekar CR, Udy AA, Boots RJ, Lipman J, Cook D. Tracheal cuff pressure monitoring in the ICU: a literature review and survey of current practice in Queensland. Anaesth Intensive Care 2014; 42: 761-70. 